\title{
Index Guiding Dependent Effects in Implant and Oxide Confined Vertical-Cavity Lasers
}

\author{
K. L. Lear, Member, IEEE, R. P. Schneider, Jr., K. D. Choquette, and S. P. Kilcoyne, Member, IEEE
}

\begin{abstract}
Implant and oxide confined vertical-cavity surfaceemitting lasers are compared in terms of properties dependent upon the nature of index guiding in the two structures including CW threshold current scaling with size, light-current linearity, pulsed operation delay, and beam profiles. The oxide confined lasers, fabricated by wet thermal oxidation, have a built-in index guide and thus exhibit substantially better properties than do lasers from the same wafer fabricated by proton implantation which rely on a thermal lens to reduce diffraction losses.
\end{abstract}

W HILE the orthogonal epitaxial layer and cavity geometry of vertical-cavity surface-emitting laser (VCSEL) diodes engenders many of their appealing properties [1], it also allows a few percent round-trip gain at most and thus restricts loss to a comparable level. This necessitates highly reflecting mirrors and small absorption, scattering, and diffraction losses. Diffraction losses in the plane-plane resonator become more dominant as device size decreases [2], necessitating the optical confinement provided by index guiding. Proton implanted, so-called gain-guided VCSEL's, have little or no structural refractive index variation laterally and thus rely on heating to a thermal lens [3]. Anomalous pulsed threshold behavior, nonlinear light-current characteristics, and varying beam profiles are all consequences of relying on thermally induced guiding. Such effects are detrimental in laser diode applications, but can be essentially eliminated by incorporating structural index guiding. For example, etched post [4], regrown [5], and oxide confined [6] VCSEL structures all have substantial lateral index variation. In this paper, we compare implant and oxide confined VCSEL's in terms of properties dependent upon the nature of index guiding: CW threshold current scaling with size, light-current linearity, modulated operation, and beam profiles.

The devices were fabricated from metalorganic vapor phase epitaxy similar to previously described designs [7] incorporating a top 19 period carbon-doped mirror and a bottom 38 period silicon-doped mirror, both with uni-parabolic grading [8]. The maximum aluminum content of the $\mathrm{Al}_{x} \mathrm{Ga}_{1-x}$ As in the mirror is $x=0.96$ except for the period closest to the one-wave cavity where it was increased to $x=0.98$ in order to enhance its wet thermal oxidation rate [9]. The fabrication of both the oxide confined [6] and the proton implanted [7]

Manuscript received August 2, 1996; revised December 27, 1995. This work was supported by the U.S. Department of Energy under Contract DEAC04-94AL85000.

The authors are with Sandia National Laboratories, Photonics Research Department 1312, MS 0603, P.O. Box 5800, Albuquerque, NM 87185 USA Publisher Item Identifier S 1041-1135(96)04330-3.

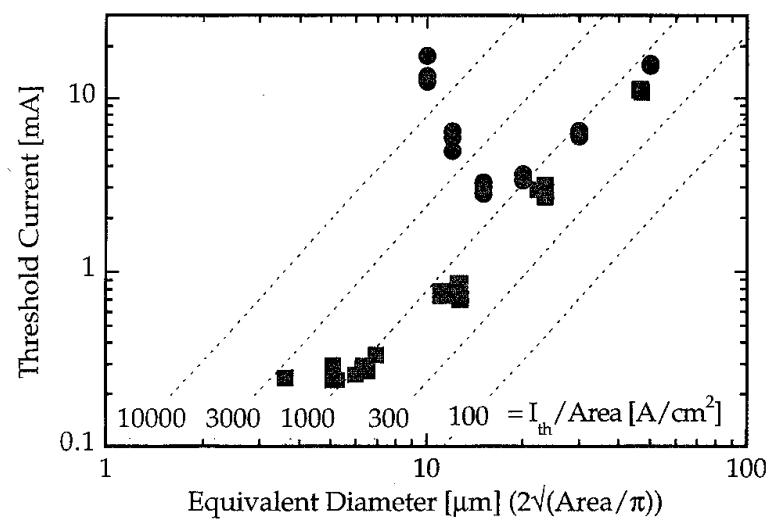

Fig. 1. Measured threshold current for circular implanted lasers (circles) and roughly square oxide confined lasers (squares) of varying size. Diagonal lines are constant effective threshold current density $\left(I_{\mathrm{th}} /\right.$ Area).

devices is described in detail elsewhere. Both types of devices presented in this paper were fabricated from the same wafer.

One of the most dramatic indications of the pathologies of implanted VCSEL's lacking index guiding is the eventual increase in $\mathrm{CW}$ threshold current with decreasing device size as seen in Fig. 1. In fact, this observation in contrast to the initial reports of record low threshold currents in small oxide confined VCSEL's by the University of Texas researchers [10] provided a major impetus for our assessment of such structures to provide index guiding. This differentiation in the threshold current scaling of implant and oxide confined VCSEL's based on identical epitaxy is clarified in the comparison of Fig. 1. While the implanted devices exhibit a minimum threshold current for device sizes of approximately $15 \mu \mathrm{m}$ diameter, the oxide confined devices appear to approach a constant threshold current for devices smaller than about $5 \mu \mathrm{m}$. It is important to note that it is the threshold current, $I_{\mathrm{th}}$, and not just the effective threshold current density $\left(I_{\mathrm{th}} /\right.$ Area) which is increasing for smaller implanted devices. Peripheral electrical losses such as edge leakage currents are proportional to the device perimeter and thus decrease for smaller devices. Increases in threshold current with decreasing device size require increases in carrier density as brought about by increased optical loss. This optical loss can be attributed to the increased penetration of the optical field into the lossy unpumped regions below the implant.

The shapes of quasi-static light-current characteristics shown in Fig. 2 further elucidate the mechanism of the threshold current increase in small implanted devices. While 


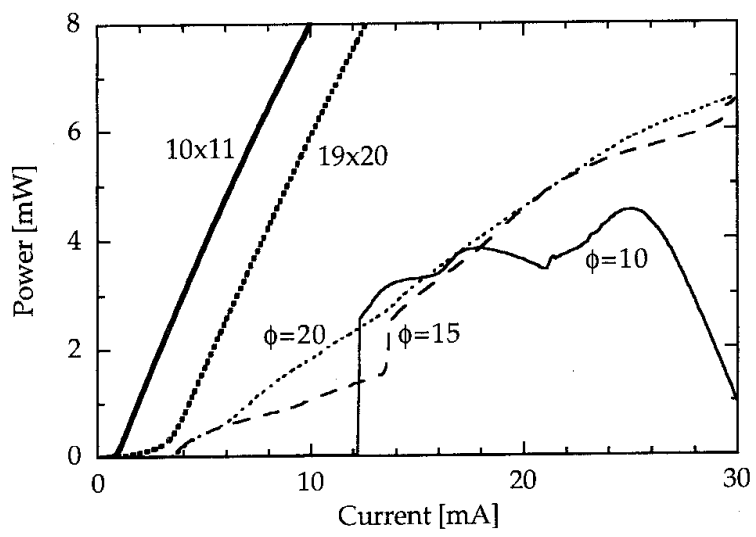

Fig. 2. Typical CW, room-temperature, light-current characteristics for circular implanted lasers (thin lines) and roughly square oxide confined lasers (thick lines) of the indicated sizes. The areas of the devices of a particular line style (e.g., dotted or solid) are comparable. The wavelength is $965 \mathrm{~nm}$ for all the lasers.

the large, $20-\mu \mathrm{m}$ diameter, implanted device has a relatively linear light-current characteristic, the medium sized $15-\mu \mathrm{m}$ diameter one begins to show a small range of increased slope efficiency immediately above threshold and strong inflections associated with additional transverse modes. Even more dramatic nonlinearities occur in the $10-\mu \mathrm{m}$ diameter device where laser output is suppressed until a large current is reached and then suddenly jumps to powers comparable to the larger devices. Approximately $1 \mathrm{~mA}$ or less of hysteresis is seen in the threshold current of the $10-\mu \mathrm{m}$ implanted laser at low frequencies. We attribute this abrupt threshold behavior to the inadequacy of the weak thermal lens to prevent diffraction losses until sufficient power is dissipated in the device. For the $10-\mu \mathrm{m}$ diameter device this amounts to $18 \mathrm{~mW}$ of input power resulting in an average active region temperature rise of $60^{\circ} \mathrm{C}$. It is ironic to note that improvements in the electrical and thermal resistance of the device due to epitaxial design [8] or heatsinking lead to reduced thermal lensing and thus inhibit the low threshold operation of small implanted devices. In contrast to these implanted devices, even the smallest oxide confined devices exhibit linear light-current curves in the vicinity of threshold with gradually reduced slope efficiencies at higher powers. Despite multimode operation over the indicated power range, no kinks are apparent since the strong index guide fixes the mode shape giving constant spatial mode-gain overlaps. Implant defined edge-emitting laser diodes sometime exhibit abrupt threshold characteristics attributed to saturable absorption that is typically accompanied by self-pulsation due to the nonlinear absorption dynamics [11]. However, none of the implanted lasers here exhibited self-pulsation at any of the $\mathrm{CW}$ or pulsed excitation conditions examined.

In order to verify that the step nature of the $10-\mu$ m diameter implanted device's light-current characteristic is due to thermal effects, the time scale of the phenomena was investigated using variable frequency modulation. Sinusoidal current modulation of $\pm 1 \mathrm{~mA}$ about a bias current slightly above the $12 \mathrm{~mA}$ threshold produced a sinusoidal light output variation for frequencies greater than $150 \mathrm{kHz}$, but resulted in abrupt

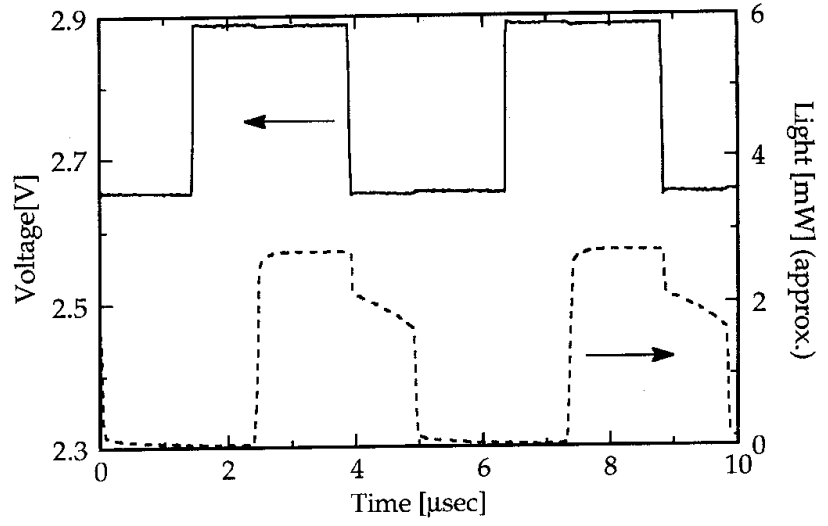

Fig. 3. Temporal response of a $10-\mu \mathrm{m}$ diameter implanted VCSEL to square wave modulation about threshold. The low- and high-voltage levels correspond to 11 and $13 \mathrm{~mA}$ respectively.

switching of the device at lower frequencies, indicating a time constant on the order of $1 \mu \mathrm{s}$. This is consistent with the diffusion of heat away from a thermal lens [3]. Other electronic or photonic mechanisms would be more rapid. For example, saturable absorption associated with implant damage [12] has relaxation times of $\sim 1 \mathrm{~ns}$ or less.

Several interesting features due to thermal lensing are seen in the results presented in Fig. 3 for a $200 \mathrm{kHz}$ square wave modulation about threshold of the $10 \mu \mathrm{m}$ diameter implanted device. The high- and low-voltage levels were set to correspond to approximately 11 and $13 \mathrm{~mA}$ into the laser. Thermal lens formation and disappearance result in long $(\sim 1$ $\mu \mathrm{s}$ ) delay times at both turn-on and turn-off. While turn-on delays have been previously studied under zero-bias pulse conditions [3], [13], the square wave modulation used here permits the examination of the turn-off dynamics as well. The electrical consequences of the approximately 2-mA decrease in drive current are seen immediately as a sudden drop in the light output at the time of the downward voltage step. The lower drive level is sufficient to sustain lasing if an existing thermal lens reduces optical loss but is insufficient to maintain a strong enough thermal lens. The power thus decays slowly during the initial portion of the low current drive and then rapidly decreases. The transition from slow to rapid power decrease may correspond to the optical mode expanding beyond the edge of the active region into the implanted region. While optical absorption due to implant damage may contribute to optical loss in this region, it cannot account for the observed behavior under the assumption of a constant optical mode profile. The turn-on and turn-off delays for the implanted devices were affected by drive levels and were shorter for larger devices. The $0.54 \mathrm{~mW}$ drop for $2 \mathrm{~mA}$ current step can be extrapolated to roughly approximate an effective threshold current of $3 \mathrm{~mA}$ if the thermal lens created at $13 \mathrm{~mA}$ could be maintained. Within the limits of the experimental apparatus $(\sim 10 \mathrm{~ns})$, no delays were observed for any of the oxide confined devices. Close inspection of the laser voltage in Fig. 3 shows a slight decrease during periods of laser operation. This likely reflects a change in the quasi fermi-levels due to an increased total carrier recombination rate in the presence of stimulated emission. 

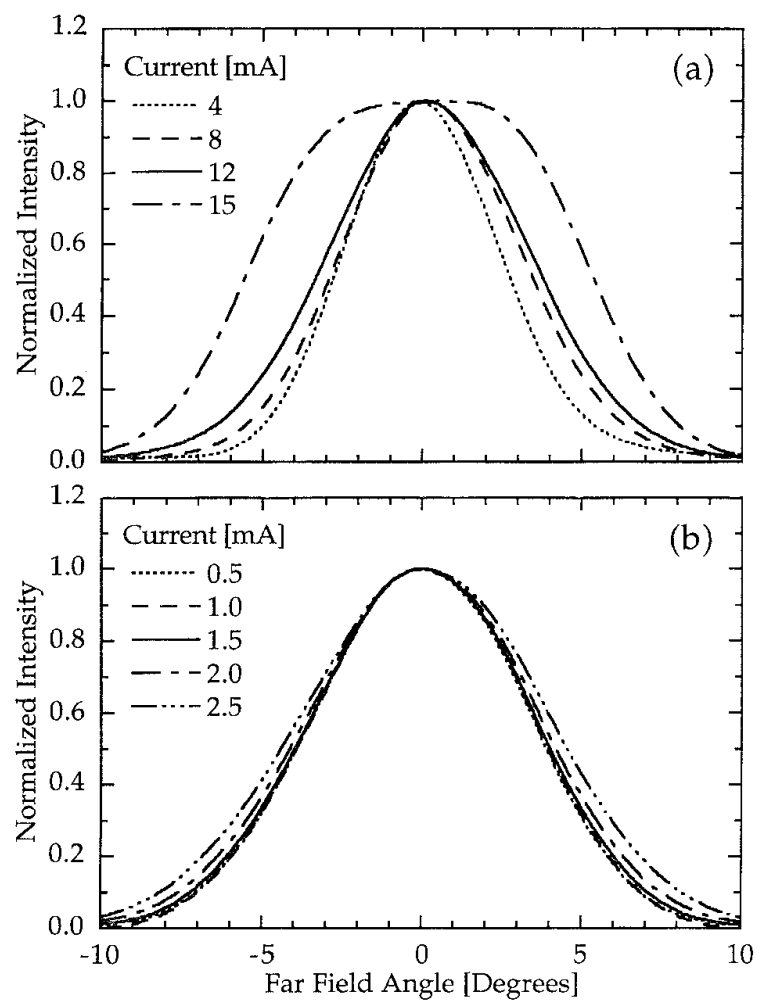

Fig. 4. Beam profiles for (a) $15 \mu \mathrm{m}$ diameter implant confined and (b) $\sim 9$ $\times 9 \mu \mathrm{m}^{2}$ oxide confined VCSEL's for various drive levels. Both lasers are single mode at the first three currents listed.

Another property influenced by the nature of index guiding in the two VCSEL structures is the beam profile. Beam profiles for a 15- $\mu \mathrm{m}$ diameter implanted VCSEL and a $\sim 9$ $\times 9 \mu \mathrm{m}^{2}$ oxide confined VCSEL are shown in Fig. 4 for a range of current levels. These sizes of devices were chosen to give a large range of single-mode operation. The implanted device had a $3 \mathrm{~mA}$ threshold and operated in a single mode up to $14 \mathrm{~mA}$ (corresponding to the kink in Fig. 2), while the oxide confined device had a $0.33 \mathrm{~mA}$ threshold and operated single-mode $(>20 \mathrm{~dB}$ transverse mode suppression [14]) up to $1.8 \mathrm{~mA}$. The beam profiles were measured with a commercial scanning slit profiler at a distance of $8 \mathrm{~mm}$ from the device. The implanted device's beam profile indicates broadening beam divergence as well as a deviation in pointing direction with increasing current in the single-mode regime. In contrast, both the width and the direction of the oxide confined VCSEL's beam are stable with increasing current throughout the single-mode regime.

An examination of properties influenced by index guiding indicate that the built-in guide in oxide confined VCSEL's is advantageous in comparison to transient thermal lensing in implanted devices. Small implant confined devices, in particular, rely on thermal lenses to prevent excessive diffraction losses. The dependence of the lens on operational and structural parameters results in increased threshold current, nonlinear light-current characteristics, long turn-on delays in pulsed operation, and unstable beam profiles. Stable beam profiles have been reported for implanted VCSEL's with spatial filtering contact apertures [15] which increase the cavity loss under the contact and thus decrease efficiency. For a given size, oxide confined VCSEL's with index steps larger than those created by thermal lensing in implanted devices are more prone to multimode operation. Approaches for improving singlemode operation in oxide confined VCSEL's including decreased effective index steps are under investigation. While modal control and other issues such as reliability and reproducibility require further investigation, the index guiding dependent properties examined in this paper as well as high efficiencies and low threshold currents make oxide confined VCSEL's attractive for a variety of applications.

\section{ACKNOWLEDGMENT}

The authors thank M. Crawford and R. Hadley for useful discussions and J. Nevers and J. Figiel for technical assistance.

\section{REFERENCES}

[1] P. L. Gourley, K. L. Lear, and R. P. Schneider, Jr., "Surface-emitting lasers," IEEE Spectrum, vol. 31, pp. 31-37, 1994.

[2] D. I. Babic, R. J. Ram, J. E. Bowers, M. Tan, and L. Yang, "Scaling laws for gain-guided vertical cavity lasers with distributed Bragg reflectors," Appl. Phys. Lett., vol. 64, pp. 1762-1764, 1994.

[3] N. K. Dutta, L. W. Tu, G. Hasnain, G. Zydzik, Y. H. Wang, and A. $\mathrm{Y}$. Cho, "Anomalous temporal response of gain guided surface emitting lasers," Electron. Lett., vol. 27, pp. 208-210, 1991.

[4] M. L. Majewski, M. G. Peters, and L. A. Coldren, "Transverse-mode spectral characteristics in index-guided vertical-cavity surface-emitting lasers," Conference on Lasers and Electro-Optics. Baltimore, MD: OSA, 1993, vol. 11, pp. 322-324.

[5] Y. A. Wu, C. J. Chang-Hasnain, and R. Nabiev, "Single mode emission from a passive-antiguide-region vertical-cavity surface-emitting laser," Electron. Lett., vol. 29, pp. 1861-1863, 1993.

[6] K. L. Lear, K. D. Choquette, R. P. Schneider Jr., and S. P. Kilcoyne, "Modal analysis of a small surface emitting laser with a selectively oxidized waveguide," Appl. Phys. Lett., vol. 66, pp. 2616-2618, 1995.

[7] K. L. Lear, R. P. Schneider, Jr., K. D. Choquette, S. P. Kilcoyne, J. J. Figiel, and J. C. Zolper, "Vertical cavity surface emitting lasers with $21 \%$ efficiency by metalorganic vapor phase epitaxy," Photon. Technol. Lett., vol. 6, pp. 1053-1055, 1994.

[8] K. L. Lear and R. P. Schneider, Jr., "Uniparabolic mirror grading for vertical cavity surface emitting lasers," Appl. Phys. Lett., vol. 68, pp. $605-607,1996$.

[9] K. D. Choquette, R. P. Schneider, Jr., K. L. Lear, and K. M. Geib, "Low threshold voltage vertical-cavity lasers fabricated by selective oxidation," Electron. Lett., vol. 30, pp. 2043-2044, 1994.

[10] D. L. Huffaker, D. G. Deppe, K. Kumar, and T. J. Rogers, "Native-oxide defined ring contact for low threshold vertical-cavity lasers," Appl. Phys. Lett., vol. 65, pp. 97-99, 1994.

[11] T. L. Paoli, "Saturable absorption effects in the self-pulsing (AlGa)As juntion laser," Appl. Phys. Lett., vol. 34, pp. 652-655, 1979.

[12] J. P. van der Ziel, W. T. Tsang, R. A. Logan, and W. M. Aug.yniak, "Pulsating output of separate confinement buried optical guide lasers due to the deliberate introduction of saturable loss," Appl. Phys. Lett., vol. 39 , pp. $376-378,1981$.

[13] G. Hasnain, K. Tai, L. Yang, Y. H. Wang, R. J. Fischer, J. D. Wynn, B. Weir, N. K. Dutta, and A. Y. Cho, "Performance of gain-guided surface emitting lasers with semiconductor distributed Bragg reflectors," IEEE J. Quantum Electron., vol. 27, pp. 1377-1385, 1991.

[14] K. L. Lear, K. D. Choquette, R. P. Schneider, Jr., S. P. Kilcoyne, and K. M. Geib, "Selectively oxidised vertical cavity surface emitting lasers with $50 \%$ power conversion efficiency," Electron. Lett., vol. 31, pp. 208-209, 1995.

[15] R. A. Morgan, G. D. Guth, M. W. Focht, M. T. Asom, K. Kojima, L. E. Rogers, and S. E. Callis, "Transverse mode control of verticalcavity top-surface-emitting lasers," Photon. Technol. Lett., vol. 4, pp. $374-377,1993$. 